$16^{\text {th }}$ International Congress of Metrology, 12006 (2013)

DOI: $10.1051 /$ metrology/201312006

(C) Owned by the authors, published by EDP Sciences, 2013

\title{
Centre For Intercomparisons
}

\author{
Azra Hajradinovic ${ }^{1 \mathrm{a}}$ and Viktoria Jonasson ${ }^{\text {la }}$ \\ ${ }^{1}$ SP Technical Research Institute of Sweden, Box 857, SE-501 15 Borås, Sweden
}

\begin{abstract}
To measure is to know" Lord Kelvin said. But how well are you measuring and do you measure in the right way? A simple way to check the quality of your measurement is to attend in intercomparisons. Participating in an intercomparison can be a simple and powerful way to verify a measurement capability and it is the most effective way to find out how well one measures. CFI is a cooperation between the NMIs (National Metrology institutes) in Nordic countries aimed at providing intercomparisons for secondary laboratories. We intend to present our experiences when offering intercomparisons world-wide and connecting with other services in Europe. Our major areas are so far: electrical, force, length, mass, pressure, vacuum and temperature but the range will be modified due to the stakeholders demands.
\end{abstract}

\section{Centre For Intercomparisons - CFI}

CFI is a cooperation between the NMIs (National Metrology institutes) in Nordic countries aimed at providing intercomparisons for secondary laboratories. SP Technical Research Institute of Sweden administrate the webpage of CFI.

We intend to present our experiences when offering intercomparisons world-wide and connecting with other services in Europe. Our major areas are: electrical, force, length, mass, pressure, vacuum and temperature. Some examples of previous intercomparisons measurements are: "Calibration of inductance", "Calibration of nonautomatic weighing instruments", "Verification and calibration of a uniaxial testing machine" etc.

So far the intercomparisons performed have been based on our knowledge of stakeholder's needs but also on direct requests from stakeholders.

\subsection{Why Intercomparisons?}

Laboratories are recommended to take part in proficiency testing schemes on a regular basis. Interlaboratory comparisons, ILC (also called intercomparison or proficiency testing, PT) are common means of determining the testing and metrological performance of a laboratory. Interlaboratory comparisons are used (e.g. by accreditation bodies, companies, metrology and testing associations) to assess the technical competence of a laboratory and the quality of measurement system (method, equipment, personnel) performance. Many standards like ISO/IEC 17025 also support the use of intercomparisons.

Intercomparisons can also be used to ensure the testing and metrological performance of a laboratory over a period of time as well as in identifying areas of improvement. It is a good way to train laboratory personnel and to compare different laboratories. The results of intercomparisons can be used to demonstrate that a company has a good technical base when discussing with business relations as well as developing laboratory uncertainties.

\subsection{Why a cooperation?}

The needs for intercomparisons are often bigger than the availability. Through cooperation between the Nordic NMIs we can offer a wider range of comparisons through one organisation. Collaboration also provides better opportunities to plan the comparisons requested by customers as well as the choice of reference laboratory or laboratories. The combined experience from the NMIs makes it easier to meet the demands from stakeholders as well as offering more flexibility. We welcome more NMIs to join us!

\subsection{How?}

Interlaboratory comparison programmes provide the organisation, performance and evaluation of calibrations or tests made on the same or similar items or materials by two or more laboratories in accordance with predetermined conditions.

\footnotetext{
${ }^{\mathrm{a}}$ Corresponding author: info@intercomparison.org
} 
Intercomparisons give the possibility of evaluating the laboratory performance under normal conditions comparing the results with a reference laboratory. The measurements are performed by the laboratory personnel in the measurement environment of the laboratory concerned. This is of great value as the results reveal relevant influences from the different elements of the laboratory's measurement system, such as the chosen measurement method, environment etc.

Ease of use is maintained though a web based solution where all handling of information and data is handled. The web site contains planned, on-going and past intercomparisons. Requests for participations, downloading of instructions and reports as well as uploading of data are handled through this site as all other communications with CFI.

Confidentiality of proficiency testing results is maintained by identifying the participants only with a unique code. The PT provider will not disclose confidential information to any third party. After signing up for an intercomparison, your company will receive a confirmation from the PT provider.

\subsection{When?}

When to take part in an intercomparison is normally up to each laboratory to plan. An accredited laboratory though has to fulfill demands according the ISO/IEC 17025 which stipulates participation in a number of quality assurance activities, including participation in intercomparisons. If there has been a major change in the laboratory's activities, introduction of a new procedure, reference equipment etc., participation in an intercomparison is normally recommended.

Historically it has been very hard for laboratories in some calibration and testing fields to make these planned comparisons since there have been too few available. The CFI (Centre For Intercomparisons) now offers a five year plan where each laboratory can plan their activities for the years to come. If no suitable intercomparisons are listed among the planned or on-going activities, you are welcome to request one in the area required.

\subsection{Our website}

Participating in an intercomparison can be a simple and powerful way to verify a measurement capability and it is the most effective way to find out how well one measures.

On our website (http://www.intercomparison.org) you can find a clear overview of the comparative measurements that presently are available in a number of areas. The website also contains a login option for participants, enabling access to files connected with intercomparisons, uploading their own results and downloading reports.
If you need an intercomparison, let us know! 